\title{
Adoption of Customer Accounting in Selected Manufacturing Firms Listed on Nigerian Stock Exchange
}

\author{
Apiti Christopher Uzoma \\ Accountancy Department, \\ University of Nigeria, Enugu, \\ Nigeria
}

\author{
Dr. Ugwoke Robinson O \\ Accountancy Department, \\ University of Nigeria, Enugu, \\ Nigeria
}

\author{
Dr. Chiekezie Njideka Rita \\ Anambra state Polytechnic, \\ Nigeria
}

\begin{abstract}
This study-examined the level of adoption of customer accounting in selected manufacturing firms listed on Nigerian Stock Exchange with the aim of establishing whether there are differences in financial performance of the firms. The study is descriptive in nature and uses survey techniques. The total population was 67 companies as listed in the Nigerian stock exchange and was reduced to 48 companies. Analysis of variance (F- Ratio) and scheffes' (fs) test were used in analysing collected data. The result of the study revealed that the mean financial performance of full adopters of CA methods was 4.74 and 11.74 greater than partial adopters and non-adopters respectively, while partial adopters' was 7.0 greater than that of non-adopters of CA methods. However, this study proves that the practice of $\mathrm{CA}$ in Nigerian manufacturing companies is still below average and the necessity to improve this situation is the current challenge.
\end{abstract}

Key words: Adoption, Customer Accounting, Financial Performance, Manufacturing Firms, Nigeria.

\section{INTRODUCTION:}

The world which is said to be a global village with an increasing competition locally and globally; and advancement in technology poses a lot of challenges to the business world. This explains the intensive dynamic changes in the business environment and increasingly unpredictable in recent decades. In recent time, managing a company has become more demanding causing companies to apply different strategies in order to gain competitive advantage. For any business to survive in this type of environment, it needs accounting information for day to day decision making in planning, organizing, directing and control. Management accounting information contributes significantly to the effective functioning of management process. Management accounting is expected to provide this information since it is aims at providing internally generated information to aid management decision making. Although a number of growing researchers in 1980s and 1990s began to recognise that management accounting was not adapting to changes in the modern business environment and as such, was not fulfilling its function to aid managers (Johnson and Koplan, 1987; Bromwich and Bhimain, 1989, 1996). The need to improve the quality of management accounting information by focusing more on non-financial information about the business environment pave way for strategic management accounting (SMA). SMA involve the generation of external accounting information which is customer focused and market driven for managers through a range of techniques and tools to facilitate strategically oriented decision making. Researchers have identified various tools of strategic management accounting that will help managers to face the recent challenges in the business world. The most acceptable SMA tools in SMA literature are the ones identify by Cadez and Guilding (2008), which are:
i) Strategic Costing.
ii) Strategic Planning, Control and Performance
management.
iii) Strategic Decision making.
iv) Competitor Accounting.
v) Customer Accounting.

Guilding \& McManus, 2002defined Customer Accounting as "all accounting practices for assessing profits, sales, or present value of earnings relating to a customer or customer group". They conceptualized customer accounting into five techniques namely (1) customer profitability analysis (CPA), (2) customer segment profitability analysis (CSPA), (3) lifetime customer profitability analysis (LCPA), (4) valuation of customer as assets (VCA) and, (5) customer accounting (the holistic notion). But, in recent studies Customer Accounting has been restructured to include three dimensions: namely (1) customer profitability analysis (CPA), (2) lifetime customer profitability analysis (LCPA), (3) valuation of customer as assets (VCA) (Cadez, 2006; Cadez \& Guilding 2008), although Hamzah \& TriDung (2016) believed that the technique should be four which include(1) customer profitability analysis (CPA), (2) lifetime customer profitability analysis (LCPA), (3) valuation of customer as assets (VCA), and (4) customer equity analysis (CEA). These techniques according to the authors are expected to meet information requirement, 
support strategic decisions and maintain organizational performance in manufacturing companies. The current research adopted the three (3) dimensions as suggested by (Cadez, 2006; Cadez \& Guilding 2008),

In every economy especially developing economy like Nigeria, the manufacturing sector is one of the most important in terms of employment creation, wealth creation and increase the standard of living of the people. In Nigeria, this sector remains weak and contributes little to GDP of the nation. Previous research shows that, from a modest $4.8 \%$ in 1960 , manufacturing contribution to GDP increased to $7.2 \%$ in 1970 and $7.4 \%$ in 1975 . In 1980 it declined to $5.4 \%$, but then surged to a record high of $10.7 \%$ in 1985. By 1990, the share of manufacturing in GDP stood at $8.1 \%$ but fell to $7.9 \%$ in $1992 ; 6.7 \%$ in 1995 and fell further to $6.3 \%$ in 1997. As at 2001 the share of manufacturing in GDP dropped to $3.4 \%$ from $6.2 \%$ in 2000. In 2013, it increased to $4.23 \%$ which is less than what it was in 1960 (CBN, 2013).According to the Nigeria GDP report by National Bureau of Statistics (NBS, 2017) the nominal GDP growth of the manufacturing sector in the third quarter of 2016 was recorded at $-2.93 \%$ (year-onyear), which is $7.73 \%$ point lower than $4.80 \%$ recorded in the corresponding period of 2015. In 2017 third quarter the Real GDP for the sector was at $-2.85 \%$. CBN, 2013 noted that the contribution of the sector to Gross Domestic Product (GDP) remains very small when compared to strong manufacturing sectors in other emerging economics, where structural changes has already occurred and where millions have been lifted out of poverty; In Countries like Brazil, China, Malaysia, Thailand and Indonesia; manufacturing companies contributes 20\%, 34\%, 30\%, $35 \%$ and $28 \%$ of its GDP respectively (Ogbu, 2012). The poor performance of the Nigerian manufacturing company can be linked to: (i) poor state of physical infrastructure, (ii) lack of funding and financial services, (iii) insufficient quality control, (iv) absence of industrial core base, (v) low investment in research and development, (vi) low level of technology, (vii) unavailability and poor flow of data/information among others, (Vision 2010 document).

Currently, the global business world is information (internal and external) oriented and any company that lacks information may not survive. Harande (2009), opine that "Progress, development, and growth of manufacturing firms largely depend on effective use of information". Muo \& Okeke (2009) in their research noted that manufacturing firms have found it difficult to key in to new business methods and technology especially information communication technology to boost operations. CA information is imperatively important and manufacturing firms in Nigeria and other developing countries should consider adopting it for better performance.

Customer accounting information usage provides organizations with customer or customer group performance and hence better understanding of how to handle customers and gain competitive advantage and maintain profitability. In Nigeria, it is imperative to say that the huge gap that exist between effective business planning and strategic decision making process in manufacturing sector may be because organizations have not fully applied customer accounting method. The argument is that adoption of customer accounting practice is more likely to lead to higher organizational performance. This is because CA is likely to provide information for strategic purposes based on customers' actions. Other researchers investigated the relationship and impact of CA methods on financial performance mostly in developed countries with little or no effort to determine the adoption level among organizations. Hence, this study seeks to fill up the gap identified in the previous studies by expanding the scope and time coverage in the Nigeria context. Based on the above premise, the study developed the following research questions and hypotheses.

\section{RESEARCH QUESTIONS}

1. To what extent do manufacturing companies adopt Customer Accounting methods for financial improvement?

2. To what extent do the various levels (full, partial and non) of adopters of CA methods differ in financial performance?

\section{RESEARCH HYPOTHESES}

1. The various levels of adopters of Customer Accounting methods differ significantly in financial performance.

\subsection{THEORETICAL FRAMEWORK}

The research is built on the foundation of agency theory and profit maximisation theory.

According to Delves and Patrick (1973) "An agency relationship is one in which "one or more persons (the principal) engage another person (the agent) to perform some service on their behalf which involves delegating some decision making authority to the agent". The theory holds a central role in the strategic management accounting literature. It describes the fundamental conflict between self-interested managers and owners, when the former have the control of the firm but the latter bear most of the wealth effects.

The manager or organizations management must seek to adopt strategies that will uplift the face of the organization financially for both parties to benefit since they are the field workers, hence, Customer accounting practice.

The profit maximizing or competition-based theory holds that profit maximization is at the forefront of every business objective. Profits maximization in this contest refer to real profits which is a surplus above the average cost of production; which could also be trace to individual or group of customers. The application of this theory to customer accounting is real since customer accounting deals with profit generated from individual customer or customer group.

\section{0}

\section{REVIEW OF RELATED LITERATURE}

2.1 Methods of Customer Accounting (CA)

Customer accounting basically is aimed at providing organization with financial and non-financial information about customer or customer group performance with a 
better understanding of how to deal with customers and gain competitive advantage and maintain profitability customer or customer group.

Although Guilding and McManus (2002) identified five CA techniques, recent studies (Cadez, 2006; Cadez \& Guilding, 2008) as stated earlier restructured CA into three dimensions, these include: (i) Customer profitability analysis (CPA), (ii) lifetime customer profitability analysis (LCPA), (iii) Valuation of customers as assets (VCA).

\subsubsection{Customer Profitability Analysis (CPA)}

Customer Profitability Analysis is a tool that identifies the cost and profit of an individual customer or group of customers. In managerial accounting, it shifts the focus from product line profitability to individual customer or group of customer's profitability. It is seen as a technique that provides management with information about the profitability of individual customers or customer groups/segment. Basically, CPA involves calculating the costs and revenues from doing business with customers (Foster and Gupta, 1994).

\subsubsection{Lifetime Customer Profitability Analysis (LCPA)}

Lifetime customer profitability analysis is a prediction of the net profit attributed to the entire future relationship with a customer. Lifetime customer profitability analysis can also be referred to as customer lifetime value (CLV or often CLTV), lifetime customer value (LCV), or life-time value (LTV). Customer lifetime value can also be defined as the naira value of a customer relationship, based on the present value of the projected future cash flows from the customer relationship. Customer lifetime value is an important concept in that it encourages firms to shift their focus from quarterly profits to the long-term health of their customer relationships. Customer lifetime value is an important number because it represents an upper limit on spending to acquire new customers.

It enables organization to find out which customers are more profitable in long-life term, so that customer relationships can be changed or controlled to increase the organization's profitability (Smith, 1993).

\subsubsection{Valuation of Customer as Assets (VCA)}

Assets are actually tools of generating economic benefits that flows into the entity over a particular period of time. This technique uses the word asset to connect to customer(s) (Foster \& Gupta (1994); \& Ward (1992). Similarly, Levitt(1983: pg.91-92) states “'a company's most precious asset is its relationship with its customers .... like any other assets, these relationships can appreciate or depreciate". The above shortcoming in accounting can not only be restricted to academics but also in accounting practice. However, Albrecht quotes Jan Carlson, the former CEO of the airline SAS, in the following manner:

"Look at our balance sheet. On the asset side, you can still see so-and-so many aircraft worth so-and-so many billions. But it's wrong; we are fooling ourselves. What we should put on the asset side is, last year SAS carried soand-so many happy passengers. Because that is the only asset we've got - people who are happy with our service and are willing to come back and pay for it once again" (1988: pg. 23).

According to Guilding and McManus, 2002, "Valuation of customers or customer groups as assets refers to the calculation of the value of customers to the company. For example, this could be undertaken by computing the present value of all future profit streams attributable to a particular customer or group of customers". Organizations/managers are beginning to understand that sometimes the huge customer database especially in this era of strong competition and technological changes may not mean that customer value is high (Day, 1994; Gale \& Wood, 1994; Woodruff, 1997). Yonggui and Hing, (2004), affirm that in this present age and future, the greatest critical element for the success of any firm is carrying a better customer value.

\subsection{The Relationship between CA Information Usage and Organizational Performance}

The aim and objective of every business is to increase organizational performance while gaining competitive advantage. Porter (1980) in an attempt to explain competitive advantage opines that "competitive advantage is at the heart of a firm's performance on competitive markets". In his words, competitive advantage means having low cost, differentiation advantage or a successful focus strategy and it grows fundamentally out of value a firm is able to create for its customers that exceeds the firms cost of creating it, invariably, competitive advantage is achieve when the revenue generated from a customer(s) because of the services rendered to him exceeds its cost. Customers accounting information provides leverage for achieving competitive advantage thereby leading to better performance. Heford (2008) assert that an organization that is effective in competitive intelligence obtains better and more complete information, enabling the organization to improve its decision-making. Furthermore, Chiekezie, (2014) opine that, firms that are more effective in their competitive intelligence effort becomes better performing organizations.

\subsection{METHODOLOGY}

The study adopted survey research which is an aspect of descriptive research design. In drawing the population element the researcher adopted the method applied by Chiekezie, Egbunike\&Odum 2014, by using four top strategic management staff namely the chief executive officer, chief accountant or Chief financial officer, marketing director, chief controller or chief planner/analyst. The total population was 67 companies as listed in the Nigerian stock exchange factbook of $2017 / 2018$ and was reduced to 48 companies using judgmental sampling techniques. The researcher employed judgmental sampling methods based on the following reasons:

1. Incomplete financial information of some companies, this led to elimination of those companies.

2. Some companies seems to be delisted from Nigeria Stock Exchange within the period under 
study, they were equally elimination from the study.

3. Few of the companies denied the researcher access to information.
The table below shows the sampling size of the study having eliminated all the companies affected by the above reasons.

Table 3.1 Sample distribution of the Manufacturing Sector.

\begin{tabular}{|l|c|}
\hline Sub-sector & No of Companies \\
\hline Consumer Goods & 16 \\
\hline Industrial Goods & 11 \\
\hline Construction/Real Estate & 3 \\
\hline Conglomerates & 5 \\
\hline Agriculture & 5 \\
\hline Oil and Gas & 8 \\
\hline TOTAL & $\mathbf{4 8}$ \\
\hline
\end{tabular}

Source: Sample distribution computed by the researcher

The research made use of both primary and secondary data; the instrument used for primary data is questionnaire while annual report/ NSE FactBook were used for secondary data. Return on Asset (ROA) was used to measure the financial performance while the responses from the 192 $(4 * 48)$ respondents are used to measure the practice of customer accounting. Analysis of Variance (F-ratio) and Scheffe's (Fs) test was used to analyse the data obtained from the sample companies while Analysis of Variance (Fratio) was used todetermine whether significant difference exist in the financial performance of the three categories of CA adopters, Scheffees (Fs) test was used to know which particular group creates the difference or is worst hit or is superior.

Measurement of the Variables of the Study

The research has two main variables, dependent and independent variables. Customer accounting is the independent variable while financial performance is the dependent variable.). A 18 - item activities involved in CA application was developed in line with Cadez, (2006); Cadez \& Guilding, (2008); Almawali, Zainuddin, \& Kader Ali, (2012), Al-Mawali \& Amoush, (2013). 7 items were used to measure CPA, another 6 items used to measure LCPA while VCA was measured by 5 items.

\section{Measurement of the rate of adoption of Customer Accounting}

To determine the adoption of CA methods, the researchers tend to use the responses from the questionnaire; which was analysed using the statistical instrument mentioned. The major question among others used to ascertain this is "To what extent does your company use the following CA methods (CPA, LCPA, VCA) for strategic decision in the last five years?" The extent of the adoption of CA methods was determined using scale mean. Scale mean is obtained by multiplying the weighted average (3) (i.e $1+2+3+4+5=$ $15 / 5=3$ ) by the number of items listed for each CA method. For Customer Profitability Analysis (CPA), the scale mean is $3^{*} 7=21$; Life time Customer Profitability Analysis (LCPA) is $3 * 6=18$; Valuation of Customer as Asset (VCA) is $3 * 5=15$. Mean score below the scale mean will be interpreted as little extent while above the scale mean will be interpreted as large extent. The adoption level will be summarized as follows

Table: 3.1 Adoption Level of Customer Accounting

\begin{tabular}{|l|l|l|l|l|l|}
\hline S/N & 1 & 2 & 3 & 4 & 5 \\
\hline Responses & Not at all & Little extent & An extent & Large extent & Very large extent \\
\hline Adoption & NON & PARTIAL & \multicolumn{3}{|c|}{ FULL ADOPTERS } \\
Level & ADOPTERS & ADOPTERS & & \\
\hline Mean score & $1.00-1.99$ & $2.00-2.99$ & \multicolumn{3}{|c|}{$3.00-5.00$} \\
\hline
\end{tabular}

Decision for Categorization of Companies into adoption levels

Decision rule for the categorization of companies into non-adopters, partial adopters and full adopters was done as follows:

CPA:

Lower limit $1.00 \times \quad 7=7$

Upper limit $1.99 \times 7=13.93$

Lower limit $2.00 \times 7=14.00$

Upper limit $2.99 \times 7=20.93$

Lower limit $3.00 \times 7=21.00$

Upper Limit $3.99 \times 7=27.93$

Lower limit $4.00 \times 7=28.00$

Upper Limit $4.99 \mathrm{x}=34.93$

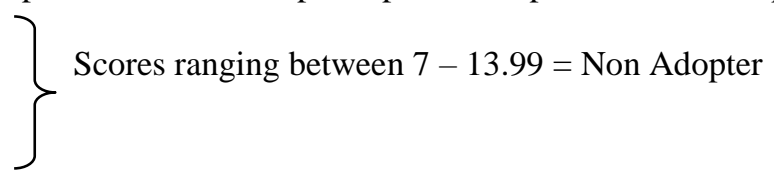

Scores ranging between $14-20.99=$ Partial Adopter

\}

Scores ranging between $21-35.00=$ full adopter 


\section{LCPA:}

Lower limit 1.00 x $6=6$

Upper limit 1.99 x $6=11.94$

Lower limit $2.00 \times 6=12.00$

Upper limit 2.99 x $6=17.94$

Lower limit 3.00 x $6=18.00$

Upper Limit $3.99 \times 6=23.94$

Lower limit 4.00 x $6=24.00$

Upper Limit 4.99 × $6=29.94$

\section{VCA:}

Lower limit $1.00 \times 5=5$

Upper limit 1.99 x $5=9.95$

Lower limit 2.00 x $5=10.00$

Upper limit 2.99 x $5=14.95$

Lower limit $3.00 \times 5=15.00$

Upper Limit $3.99 \times 5=23.95$

Lower limit $4.00 \times 5=20.00$

Upper Limit $4.99 \times 5=24.95$

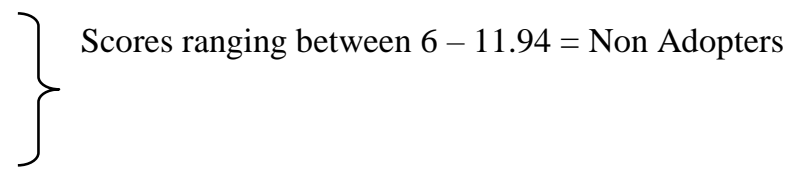

Scores ranging between $12-17.99=$ Partial Adopters<smiles>CCCC</smiles>

Scores ranging between $21-30.00=$ full adopters

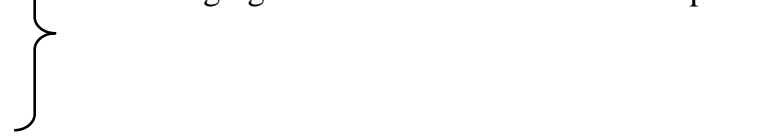

Scores ranging between $5-9.99=$ Non Adoption

Scores ranging between $12-14.99=$ Partial Adoption<smiles>[CH]CC</smiles>

Scores ranging between $15-25.00 .=$ full adoption \}

Determination of non- adopters, partial adopters and full adopters of all CA methods

Using the categorization of adoption of each CA method, companies' adoption of all CA methods was done as follows:

Non-adoption + Non-adoption + Non-adoption $=$ Non adopter

Non-adoption + Non-adoption + Partial adoption $=$ Non adopter

Non-adoption + Partial Adoption + full adoption $=$ Partial adopter Partial adoption + Full Adoption + Full Adoption $=$ Partial Adopter Partial adoption + Partial Adoption + Partial Adoption $=$ Partial Adopter

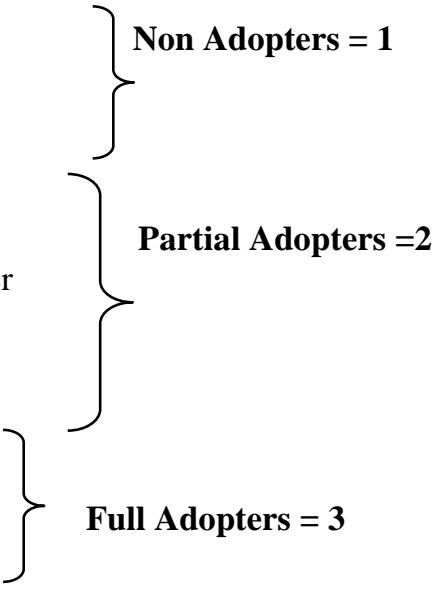

company for the last five years was obtained and subjected to analysis using the formula;

\section{ROA $=\underline{\text { Profit before Taxes }}$}

Total Assets

At the end, a five year average ROA was computed for each company. return on assets (ROA) which is an accounting based measure of return. The financial statement for each 


\begin{tabular}{|c|c|c|c|c|c|c|c|c|c|c|c|c|c|c|c|c|}
\hline \multirow[b]{2}{*}{$\begin{array}{l}\text { CATEGO } \\
\text { RIES }\end{array}$} & \multirow[b]{2}{*}{ COMPANY } & \multicolumn{3}{|l|}{2013} & \multicolumn{3}{|l|}{2014} & \multicolumn{3}{|l|}{2015} & \multicolumn{3}{|l|}{2016} & \multicolumn{3}{|l|}{2017} \\
\hline & & $\begin{array}{l}\text { PROFIT } \\
\text { N'000 } \\
\end{array}$ & $\begin{array}{l}\text { ASSET } \\
\text { N'000 } \\
\end{array}$ & $\begin{array}{l}\mathrm{R} \\
\mathrm{O} \\
\mathrm{A}\end{array}$ & $\begin{array}{l}\text { PROFIT } \\
\text { N'000 }\end{array}$ & $\begin{array}{l}\text { ASSET } \\
\mathrm{N}^{\prime} 000 \\
\end{array}$ & $\begin{array}{l}\mathrm{R} \\
\mathrm{O} \\
\mathrm{A}\end{array}$ & $\begin{array}{l}\text { PROFIT } \\
\text { N'000 }\end{array}$ & $\begin{array}{l}\text { ASSET } \\
N^{\prime} 000 \\
\end{array}$ & $\begin{array}{l}\mathrm{R} \\
\mathrm{O} \\
\mathrm{A}\end{array}$ & $\begin{array}{l}\text { PROFIT } \\
\text { N'000 }\end{array}$ & $\begin{array}{l}\text { ASSET } \\
\mathrm{N}^{\prime} 000 \\
\end{array}$ & $\begin{array}{l}\mathrm{R} \\
\mathrm{O} \\
\mathrm{A}\end{array}$ & $\begin{array}{l}\text { PROFIT } \\
\text { N'}^{\prime} 000 \\
\end{array}$ & $\begin{array}{l}\text { ASSET } \\
\text { N'000 }\end{array}$ & ROA \\
\hline $\begin{array}{l}\text { CONSUM } \\
\text { ER } \\
\text { GOODS }\end{array}$ & $\begin{array}{l}\text { PZ } \\
\text { CUSSONS } \\
\text { NIG PLC }\end{array}$ & $\begin{array}{l}7,650,265.0 \\
0\end{array}$ & $\begin{array}{l}72,296,420 . \\
00\end{array}$ & $\begin{array}{l}11 \\
\%\end{array}$ & $\begin{array}{l}6,949,985.0 \\
0\end{array}$ & $\begin{array}{l}70,965,735 . \\
00\end{array}$ & $\begin{array}{l}10 \\
\%\end{array}$ & $\begin{array}{l}6,556,814 . \\
00\end{array}$ & $\begin{array}{l}67,387,91 \\
4.00\end{array}$ & $\begin{array}{l}10 \\
\%\end{array}$ & $\begin{array}{l}3,148,196 . \\
00\end{array}$ & $\begin{array}{l}74,430,174 . \\
00\end{array}$ & $\begin{array}{l}4 \\
\%\end{array}$ & $\begin{array}{l}4,811,169 . \\
00\end{array}$ & $\begin{array}{l}90,087,52 \\
5.00\end{array}$ & $5 \%$ \\
\hline & $\begin{array}{l}\text { DANDOTE } \\
\text { FLOUR } \\
\text { MILLS OLC }\end{array}$ & $\begin{array}{l}- \\
8,342,294.0 \\
0\end{array}$ & $\begin{array}{l}75,481,545 . \\
00\end{array}$ & $\begin{array}{l}- \\
11 \\
\%\end{array}$ & $\begin{array}{l} \\
9,258,013.0 \\
0\end{array}$ & $\begin{array}{l}54,801,489 . \\
00\end{array}$ & $\begin{array}{l}- \\
17 \\
\%\end{array}$ & $\begin{array}{l} \\
12,466,20 \\
8.00\end{array}$ & $\begin{array}{l}49,354,98 \\
2.00\end{array}$ & $\begin{array}{l}- \\
25 \\
\%\end{array}$ & $\begin{array}{l}11,818,96 \\
6.00\end{array}$ & $\begin{array}{l}78,979,982 . \\
00\end{array}$ & $\begin{array}{l}15 \\
\%\end{array}$ & $\begin{array}{l}22,439,87 \\
1.00\end{array}$ & $\begin{array}{l}129,357,1 \\
18.00\end{array}$ & $17 \%$ \\
\hline & $\begin{array}{l}\text { VITAFOAM } \\
\text { NIG PLC }\end{array}$ & $614,162.00$ & $\begin{array}{l}9,376,225.0 \\
0\end{array}$ & $\begin{array}{l}7 \\
\% \\
\end{array}$ & $926,312.00$ & $\begin{array}{l}11,032,131 . \\
00\end{array}$ & $\begin{array}{l}8 \\
\% \\
\end{array}$ & $\begin{array}{l}489,456.0 \\
0\end{array}$ & $\begin{array}{l}11,734,73 \\
9.00\end{array}$ & $\begin{array}{l}4 \\
\% \\
\end{array}$ & $\begin{array}{l}522,757.0 \\
0\end{array}$ & $\begin{array}{l}13,022,584 . \\
00\end{array}$ & $\begin{array}{l}4 \\
\% \\
\end{array}$ & $\begin{array}{l}290,280.0 \\
0\end{array}$ & $\begin{array}{l}12,974,51 \\
3.00\end{array}$ & $2 \%$ \\
\hline & $\begin{array}{l}\text { DANGOTE } \\
\text { SUGAR } \\
\text { REFINERY } \\
\text { PLC } \\
\end{array}$ & $\begin{array}{l}16,265,159 . \\
00\end{array}$ & $\begin{array}{l}83,159,878 . \\
00\end{array}$ & $\begin{array}{l}20 \\
\%\end{array}$ & $\begin{array}{l}15,273,152 . \\
00\end{array}$ & $\begin{array}{l}92,801,302 . \\
00\end{array}$ & $\begin{array}{l}16 \\
\%\end{array}$ & $\begin{array}{l}16,155,60 \\
9.00\end{array}$ & $\begin{array}{l}102,232,1 \\
44.00\end{array}$ & $\begin{array}{l}16 \\
\%\end{array}$ & $\begin{array}{l}19,614,43 \\
4.00\end{array}$ & $\begin{array}{l}172,169,458 \\
.00\end{array}$ & $\begin{array}{l}11 \\
\%\end{array}$ & $\begin{array}{l}53,598,86 \\
8.00\end{array}$ & $\begin{array}{l}195,080,4 \\
49.00\end{array}$ & $27 \%$ \\
\hline & $\begin{array}{l}\text { UNILEVER } \\
\text { NIG PLC }\end{array}$ & $\begin{array}{l}6,793,615.0 \\
0 \\
\end{array}$ & $\begin{array}{l}43,754,114 . \\
00\end{array}$ & $\begin{array}{l}16 \\
\% \\
\end{array}$ & $\begin{array}{l}2,873,235.0 \\
0\end{array}$ & $\begin{array}{l}45,736,255 . \\
00\end{array}$ & $\begin{array}{l}6 \\
\% \\
\end{array}$ & $\begin{array}{l}1,771,063 . \\
00\end{array}$ & $\begin{array}{l}50,172,48 \\
4.00\end{array}$ & $\begin{array}{l}4 \\
\% \\
\end{array}$ & $\begin{array}{l}4,106,472 . \\
00\end{array}$ & $\begin{array}{l}72,491,309 . \\
00\end{array}$ & $\begin{array}{l}6 \\
\% \\
\end{array}$ & $\begin{array}{l}11,207,21 \\
3.00\end{array}$ & $\begin{array}{l}121,084,3 \\
65.00\end{array}$ & $9 \%$ \\
\hline & $\begin{array}{l}\text { HONEYWE } \\
\text { LL FLOUR } \\
\text { MILLS PLC }\end{array}$ & $\begin{array}{l}3,814,599.0 \\
0\end{array}$ & $\begin{array}{l}55,437,478 . \\
00\end{array}$ & $\begin{array}{l}7 \\
\%\end{array}$ & $\begin{array}{l}4,267,432.0 \\
0\end{array}$ & $\begin{array}{l}63,830,439 . \\
00\end{array}$ & $\begin{array}{l}7 \\
\%\end{array}$ & $\begin{array}{l}1,434,828 . \\
00\end{array}$ & $\begin{array}{l}67,943,44 \\
4.00\end{array}$ & $\begin{array}{l}2 \\
\%\end{array}$ & $\begin{array}{l}- \\
2,869,342 . \\
00\end{array}$ & $\begin{array}{l}76,046,576 . \\
00\end{array}$ & $\begin{array}{l}- \\
4 \\
\%\end{array}$ & $\begin{array}{l}5,469,833 . \\
00\end{array}$ & $\begin{array}{l}113,151,7 \\
15.00\end{array}$ & $5 \%$ \\
\hline & $\begin{array}{l}\text { CARBURY } \\
\text { NIG PLC }\end{array}$ & $\begin{array}{l}8,278,526.0 \\
0\end{array}$ & $\begin{array}{l}43,172,624 . \\
00\end{array}$ & $\begin{array}{l}19 \\
\%\end{array}$ & $\begin{array}{l}2,385,891.0 \\
0\end{array}$ & $\begin{array}{l}28,811,286 . \\
00\end{array}$ & $\begin{array}{l}8 \\
\% \\
\end{array}$ & $\begin{array}{l}1,577,412 . \\
00\end{array}$ & $\begin{array}{l}28,417,00 \\
5.00\end{array}$ & $\begin{array}{l}6 \\
\% \\
\end{array}$ & $\begin{array}{l}- \\
562,870.0 \\
0\end{array}$ & $\begin{array}{l}28,409,000 . \\
00\end{array}$ & $\begin{array}{l}2 \\
\%\end{array}$ & $\begin{array}{l}350,317.0 \\
0\end{array}$ & $\begin{array}{l}28,423,12 \\
2.00\end{array}$ & $1 \%$ \\
\hline & $\begin{array}{l}\text { FLOUR } \\
\text { MILLS OF } \\
\text { NIG PLC }\end{array}$ & $\begin{array}{l}11,803,161 . \\
00\end{array}$ & $\begin{array}{l}280,137,952 \\
.00\end{array}$ & $\begin{array}{l}4 \\
\% \\
\end{array}$ & $\begin{array}{l}7,686,943.0 \\
0\end{array}$ & $\begin{array}{l}297,249,445 \\
.00\end{array}$ & $\begin{array}{l}3 \\
\% \\
\end{array}$ & $\begin{array}{l}7,724,770 . \\
00\end{array}$ & $\begin{array}{l}343,260,5 \\
65.00\end{array}$ & $\begin{array}{l}2 \\
\%\end{array}$ & $\begin{array}{l}11,489,27 \\
8.00\end{array}$ & $\begin{array}{l}345,348,326 \\
.00\end{array}$ & $\begin{array}{l}3 \\
\% \\
\end{array}$ & $\begin{array}{l}10,472,84 \\
7.00\end{array}$ & $\begin{array}{l}482,603,2 \\
57.00\end{array}$ & $2 \%$ \\
\hline & $\begin{array}{l}\text { NESTLE } \\
\text { NIG PLC }\end{array}$ & $\begin{array}{l}26,047,590 . \\
00\end{array}$ & $\begin{array}{l}108,207,480 \\
.00\end{array}$ & $\begin{array}{l}24 \\
\%\end{array}$ & $\begin{array}{l}24,445,978 . \\
00\end{array}$ & $\begin{array}{l}106,062,067 \\
.00\end{array}$ & $\begin{array}{l}23 \\
\%\end{array}$ & $\begin{array}{l}29,322,47 \\
7.00\end{array}$ & $\begin{array}{l}119,215,0 \\
53.00\end{array}$ & $\begin{array}{l}25 \\
\%\end{array}$ & $\begin{array}{l}21,548,40 \\
8.00\end{array}$ & $\begin{array}{l}169,585,932 \\
.00\end{array}$ & $\begin{array}{l}13 \\
\%\end{array}$ & $\begin{array}{l}46,828,68 \\
2.00\end{array}$ & $\begin{array}{l}146,804,1 \\
28.00\end{array}$ & $32 \%$ \\
\hline & $\begin{array}{l}\text { NIGERIAN } \\
\text { BREWERIE } \\
\text { S PLC } \\
\end{array}$ & $\begin{array}{l}62,240,317 . \\
00\end{array}$ & $\begin{array}{l}252,759,633 \\
.00\end{array}$ & $\begin{array}{l}25 \\
\% \\
\end{array}$ & $\begin{array}{l}61,461,822 . \\
00\end{array}$ & $\begin{array}{l}349,229,163 \\
.00\end{array}$ & $\begin{array}{l}18 \\
\%\end{array}$ & $\begin{array}{l}54,514,97 \\
3.00\end{array}$ & $\begin{array}{l}356,218,6 \\
76.00\end{array}$ & $\begin{array}{l}15 \\
\%\end{array}$ & $\begin{array}{l}39,674,51 \\
8.00\end{array}$ & $\begin{array}{l}367,146,468 \\
.00\end{array}$ & $\begin{array}{l}11 \\
\%\end{array}$ & $\begin{array}{l}46,630,05 \\
8.00\end{array}$ & $\begin{array}{l}382,228,0 \\
93.00\end{array}$ & $12 \%$ \\
\hline & $\begin{array}{l}\text { NASCON } \\
\text { ALLIED } \\
\text { INDUSTRY } \\
\text { PLC } \\
\end{array}$ & $\begin{array}{l}4,038,405.0 \\
0\end{array}$ & $\begin{array}{l}11,431,168 . \\
00\end{array}$ & $\begin{array}{l}35 \\
\%\end{array}$ & $\begin{array}{l}2,856,399.0 \\
0\end{array}$ & $\begin{array}{l}12,555,885 . \\
00\end{array}$ & $\begin{array}{l}23 \\
\%\end{array}$ & $\begin{array}{l}3,017,564 . \\
00\end{array}$ & $\begin{array}{l}16,794,82 \\
6.00\end{array}$ & $\begin{array}{l}18 \\
\%\end{array}$ & $\begin{array}{l}2,592,772 . \\
00\end{array}$ & $\begin{array}{l}25,138,578 . \\
00\end{array}$ & $\begin{array}{l}10 \\
\%\end{array}$ & $\begin{array}{l}5,962,069 . \\
00\end{array}$ & $\begin{array}{l}26,837,93 \\
1.00\end{array}$ & $22 \%$ \\
\hline
\end{tabular}




\begin{tabular}{|c|c|c|c|c|c|c|c|c|c|c|c|c|c|c|c|c|}
\hline & $\begin{array}{l}\text { UNION } \\
\text { DICON } \\
\text { SALT PLC }\end{array}$ & $12,104.00$ & $86,427.00$ & $\begin{array}{l}14 \\
\%\end{array}$ & $\begin{array}{l}- \\
87,326.00\end{array}$ & $93,945.00$ & $\begin{array}{l}93 \\
\% \\
\end{array}$ & $\overline{-}, 285.00$ & $68,477.00$ & $\begin{array}{l}3 \\
\% \\
\end{array}$ & $\begin{array}{l}398,962.0 \\
0\end{array}$ & $119,871.00$ & $\begin{array}{l}33 \\
3 \\
\% \\
\end{array}$ & $\begin{array}{l}- \\
83,433.00 \\
\end{array}$ & $84,923.00$ & $-98 \%$ \\
\hline & $\begin{array}{l}\text { CHAMPION } \\
\text { BREWERIE } \\
\text { S PLC }\end{array}$ & $\begin{array}{l}- \\
1,730,432.0 \\
0\end{array}$ & $\begin{array}{l}9,137,716.0 \\
0\end{array}$ & $\begin{array}{l}- \\
19 \\
\%\end{array}$ & $\begin{array}{l}- \\
1,061,783.0 \\
0\end{array}$ & $\begin{array}{l}9,592,381.0 \\
0\end{array}$ & $\begin{array}{l}- \\
11 \\
\%\end{array}$ & $\begin{array}{l}248,443.0 \\
0\end{array}$ & $\begin{array}{l}10,329,16 \\
0.00\end{array}$ & $\begin{array}{l}2 \\
\%\end{array}$ & $\begin{array}{l}681,284.0 \\
0\end{array}$ & $\begin{array}{l}9,961,240.0 \\
0\end{array}$ & $\begin{array}{l}7 \\
\%\end{array}$ & $\begin{array}{l}648,242.0 \\
0\end{array}$ & $\begin{array}{l}10,088,86 \\
1.00\end{array}$ & $6 \%$ \\
\hline & $\begin{array}{l}\text { DN TRYRE } \\
\text { \& RUBBER } \\
\text { PLC }\end{array}$ & $460,432.00$ & $\begin{array}{l}2,066,906.0 \\
0\end{array}$ & $\begin{array}{l}22 \\
\%\end{array}$ & $\begin{array}{l}- \\
689,733.00\end{array}$ & $\begin{array}{l}1,949,883.0 \\
0\end{array}$ & $\begin{array}{l}- \\
35 \\
\%\end{array}$ & $\begin{array}{l}513,796.0 \\
0\end{array}$ & $\begin{array}{l}1,906,917 . \\
00\end{array}$ & $\begin{array}{l}27 \\
\%\end{array}$ & $\begin{array}{l}- \\
139,863.0 \\
0\end{array}$ & $\begin{array}{l}1,672,213.0 \\
0\end{array}$ & $\begin{array}{l}- \\
8 \\
\%\end{array}$ & $77,915.00$ & $\begin{array}{l}1,658,450 . \\
00\end{array}$ & $5 \%$ \\
\hline & $\begin{array}{l}\text { INTERNATI } \\
\text { ONAL } \\
\text { BREWERIE } \\
\text { S PLC } \\
\end{array}$ & $\begin{array}{l}3,555,546.0 \\
0\end{array}$ & $\begin{array}{l}23,036,762 . \\
00\end{array}$ & $\begin{array}{l}15 \\
\%\end{array}$ & $\begin{array}{l}3,925,500.0 \\
0\end{array}$ & $\begin{array}{l}24,370,540 . \\
00\end{array}$ & $\begin{array}{l}16 \\
\%\end{array}$ & $\begin{array}{l}2,815,554 . \\
00\end{array}$ & $\begin{array}{l}30,171,59 \\
0.00\end{array}$ & $\begin{array}{l}9 \\
\%\end{array}$ & $\begin{array}{l}3,656,826 . \\
00\end{array}$ & $\begin{array}{l}33,482,106 . \\
00\end{array}$ & $\begin{array}{l}11 \\
\%\end{array}$ & $\begin{array}{l}2,891,749 . \\
00\end{array}$ & $\begin{array}{l}44,962,73 \\
5.00\end{array}$ & $6 \%$ \\
\hline & $\begin{array}{l}\text { GUINNESS } \\
\text { NIG PLC }\end{array}$ & $\begin{array}{l}17,008,875 . \\
00\end{array}$ & $\begin{array}{l}121,060,620 \\
.00\end{array}$ & $\begin{array}{l}14 \\
\%\end{array}$ & $\begin{array}{l}11,681,560 . \\
00\end{array}$ & $\begin{array}{l}132,328,273 \\
.00\end{array}$ & $\begin{array}{l}9 \\
\%\end{array}$ & $\begin{array}{l}10,795,10 \\
2.00\end{array}$ & $\begin{array}{l}122,246,6 \\
32.00\end{array}$ & $\begin{array}{l}9 \\
\%\end{array}$ & $\begin{array}{l} \\
2,347,241 . \\
00\end{array}$ & $\begin{array}{l}136,992,444 \\
.00\end{array}$ & $\begin{array}{l} \\
2 \\
\%\end{array}$ & $\begin{array}{l}2,662,081 . \\
00\end{array}$ & $\begin{array}{l}146,038,2 \\
16.00\end{array}$ & $2 \%$ \\
\hline $\begin{array}{l}\text { INDUSTR } \\
\text { IAL } \\
\text { GOODS }\end{array}$ & $\begin{array}{l}\text { BERGER } \\
\text { PAINTS }\end{array}$ & $342,767.00$ & $\begin{array}{l}3,627,598.0 \\
0\end{array}$ & $\begin{array}{l}9 \\
\%\end{array}$ & $249,258.00$ & $\begin{array}{l}3,640,145.0 \\
0\end{array}$ & $\begin{array}{l}7 \\
\%\end{array}$ & $\begin{array}{l}565,212.0 \\
0\end{array}$ & $\begin{array}{l}3,895,870 . \\
00\end{array}$ & $\begin{array}{l}15 \\
\%\end{array}$ & $\begin{array}{l}271,770.0 \\
0\end{array}$ & $\begin{array}{l}4,102,265.0 \\
0\end{array}$ & $\begin{array}{l}7 \\
\%\end{array}$ & $\begin{array}{l}339,456.0 \\
0\end{array}$ & $\begin{array}{l}4,311,424 . \\
00\end{array}$ & $8 \%$ \\
\hline & $\begin{array}{l}\text { CHEMICAL } \\
\text { AND } \\
\text { ALLIED } \\
\text { PRODUCT }\end{array}$ & $\begin{array}{l}2,086,993.0 \\
0\end{array}$ & $\begin{array}{l}3,035,012.0 \\
0\end{array}$ & $\begin{array}{l}69 \\
\%\end{array}$ & $\begin{array}{l}2,442,140.0 \\
0\end{array}$ & $\begin{array}{l}3,080,881.0 \\
0\end{array}$ & $\begin{array}{l}79 \\
\%\end{array}$ & $\begin{array}{l}2,570,021 . \\
00\end{array}$ & $\begin{array}{l}3,409,300 . \\
00\end{array}$ & $\begin{array}{l}75 \\
\%\end{array}$ & $\begin{array}{l}2,296,821 . \\
00\end{array}$ & $\begin{array}{l}4,915,999.0 \\
0\end{array}$ & $\begin{array}{l}47 \\
\%\end{array}$ & $\begin{array}{l}2,181,711 . \\
00\end{array}$ & $\begin{array}{l}5,013,990 . \\
00\end{array}$ & $44 \%$ \\
\hline & $\begin{array}{l}\text { CEMENT } \\
\text { COMPANY } \\
\text { OF } \\
\text { NORTHERN } \\
\text { NIG PLC } \\
\end{array}$ & $\begin{array}{l}2,105,835.0 \\
0\end{array}$ & $\begin{array}{l}15,058,476 . \\
00\end{array}$ & $\begin{array}{l}14 \\
\%\end{array}$ & $\begin{array}{l}2,476,772.0 \\
0\end{array}$ & $\begin{array}{l}15,780,012 . \\
00\end{array}$ & $\begin{array}{l}16 \\
\%\end{array}$ & $\begin{array}{l}1,549,597 . \\
00\end{array}$ & $\begin{array}{l}17,146,88 \\
3.00\end{array}$ & $\begin{array}{l}9 \\
\%\end{array}$ & $\begin{array}{l}1,740,522 . \\
00\end{array}$ & $\begin{array}{l}20,030,222 . \\
00\end{array}$ & $\begin{array}{l}9 \\
\%\end{array}$ & $\begin{array}{l}4,203,153 . \\
00\end{array}$ & $\begin{array}{l}24,648,67 \\
6.00\end{array}$ & $17 \%$ \\
\hline & $\begin{array}{l}\text { FIRST } \\
\text { ALUMINIU } \\
\text { M NIG PLC }\end{array}$ & $27,714.00$ & $\begin{array}{l}8,570,793.0 \\
0\end{array}$ & $\begin{array}{l}0 \\
\% \\
\end{array}$ & $104,450.00$ & $\begin{array}{l}8,476,055.0 \\
0\end{array}$ & $\begin{array}{l}1 \\
\% \\
\end{array}$ & $41,265.00$ & $\begin{array}{l}8,237,088 . \\
00\end{array}$ & $\begin{array}{l}1 \\
\% \\
\end{array}$ & $\begin{array}{l}269,713.0 \\
0\end{array}$ & $\begin{array}{l}9,337,115.0 \\
0\end{array}$ & $\begin{array}{l}3 \\
\% \\
\end{array}$ & $\begin{array}{l}521,710.0 \\
0\end{array}$ & $\begin{array}{l}9,884,593 . \\
00\end{array}$ & $5 \%$ \\
\hline & MEYER PLC & $51,189.00$ & $\begin{array}{l}2,627,559.0 \\
0\end{array}$ & $\begin{array}{l}2 \\
\%\end{array}$ & $\begin{array}{l}- \\
37,362.00 \\
\end{array}$ & $\begin{array}{l}2,462,578.0 \\
0\end{array}$ & $\begin{array}{l} \\
2 \\
\%\end{array}$ & $60,459.00$ & $\begin{array}{l}2,328,334 . \\
00\end{array}$ & $\begin{array}{l}3 \\
\% \\
\end{array}$ & $\begin{array}{l}- \\
215,832.0 \\
0 \\
\end{array}$ & $\begin{array}{l}1,950,978.0 \\
0\end{array}$ & $\begin{array}{l}- \\
11 \\
\%\end{array}$ & $\begin{array}{l}- \\
264,809.0 \\
0 \\
\end{array}$ & $\begin{array}{l}1,917,776 . \\
00\end{array}$ & $-14 \%$ \\
\hline & $\begin{array}{l}\text { PAINTS } \\
\text { AND } \\
\text { COATINGS } \\
\text { MANUFACT } \\
\text { URERS NIG } \\
\text { PLC } \\
\end{array}$ & $292,460.00$ & $\begin{array}{l}2,337,220.0 \\
0\end{array}$ & $\begin{array}{l}13 \\
\%\end{array}$ & $215,831.00$ & $\begin{array}{l}3,334,117.0 \\
0\end{array}$ & $\begin{array}{l}6 \\
\%\end{array}$ & $\begin{array}{l}135,354.0 \\
0\end{array}$ & $\begin{array}{l}2,315,817 . \\
00\end{array}$ & $\begin{array}{l}6 \\
\%\end{array}$ & $40,139.00$ & $\begin{array}{l}2,440,618.0 \\
0\end{array}$ & $\begin{array}{l}2 \\
\%\end{array}$ & $44,210.00$ & $\begin{array}{l}2,657,365 . \\
00\end{array}$ & $2 \%$ \\
\hline & $\begin{array}{l}\text { PORTLAND } \\
\text { PAINTS } \\
\text { AND } \\
\text { PRODUCTS } \\
\text { NIG PLC }\end{array}$ & $73,646.00$ & $\begin{array}{l}2,073,222.0 \\
0\end{array}$ & $\begin{array}{l}4 \\
\%\end{array}$ & $194,297.00$ & $\begin{array}{l}2,277,558.0 \\
0\end{array}$ & $\begin{array}{l}9 \\
\%\end{array}$ & $\begin{array}{l}- \\
258,369.0 \\
0\end{array}$ & $\begin{array}{l}1,899,281 . \\
00\end{array}$ & $\begin{array}{l}14 \\
\%\end{array}$ & $7,502.00$ & $\begin{array}{l}1,754,321.0 \\
0\end{array}$ & $\begin{array}{l}0 \\
\%\end{array}$ & $\begin{array}{l}123,868.0 \\
0\end{array}$ & $\begin{array}{l}2,035,902 . \\
00\end{array}$ & $6 \%$ \\
\hline & $\begin{array}{l}\text { PREMIER } \\
\text { PAINTS PLC }\end{array}$ & $\begin{array}{l}- \\
16,002.00\end{array}$ & $285,772.00$ & $\overline{6}$ & $11,676.00$ & $288,982.00$ & $\begin{array}{l}4 \\
\%\end{array}$ & $\begin{array}{l}50,841.00 \\
\end{array}$ & $341,288.0$ & $\overline{15}$ & $32,242.00$ & $320,041.00$ & - & $\begin{array}{l}76,396.00 \\
\end{array}$ & $284,085.0$ & $-27 \%$ \\
\hline
\end{tabular}




\begin{tabular}{|c|c|c|c|c|c|c|c|c|c|c|c|c|c|c|c|c|}
\hline & & & & $\%$ & & & & & 0 & $\%$ & & & $\%$ & & 0 & \\
\hline & CUTIX PLC & $229,287.00$ & $\begin{array}{l}1,073,866.0 \\
0\end{array}$ & $\begin{array}{l}21 \\
\% \\
\end{array}$ & $264,837.00$ & $\begin{array}{l}1,744,670.0 \\
0\end{array}$ & $\begin{array}{l}15 \\
\% \\
\end{array}$ & $\begin{array}{l}202,107.0 \\
0\end{array}$ & $\begin{array}{l}1,968,814 . \\
00\end{array}$ & $\begin{array}{l}10 \\
\%\end{array}$ & $\begin{array}{l}278,114.0 \\
0\end{array}$ & $\begin{array}{l}1,891,718.0 \\
0\end{array}$ & $\begin{array}{l}15 \\
\%\end{array}$ & $\begin{array}{l}370,143.0 \\
0\end{array}$ & $\begin{array}{l}2,329,792 . \\
00\end{array}$ & $16 \%$ \\
\hline & $\begin{array}{l}\text { BETA } \\
\text { GLASS PLC }\end{array}$ & $\begin{array}{l}2,052,193.0 \\
0\end{array}$ & $\begin{array}{l}27,166,481 . \\
00\end{array}$ & $\begin{array}{l}8 \\
\%\end{array}$ & $\begin{array}{l}3,340,660.0 \\
0\end{array}$ & $\begin{array}{l}26,928,387 . \\
00\end{array}$ & $\begin{array}{l}12 \\
\%\end{array}$ & $\begin{array}{l}3,114,795 . \\
00\end{array}$ & $\begin{array}{l}27,171,06 \\
9.00\end{array}$ & $\begin{array}{l}11 \\
\%\end{array}$ & $\begin{array}{l}5,215,253 . \\
00\end{array}$ & $\begin{array}{l}33,190,672 . \\
00\end{array}$ & $\begin{array}{l}16 \\
\%\end{array}$ & $\begin{array}{l}5,854,740 . \\
00\end{array}$ & $\begin{array}{l}38,211,61 \\
3.00\end{array}$ & $15 \%$ \\
\hline & $\begin{array}{l}\text { GREIF NIG } \\
\text { PLC }\end{array}$ & $52,469.00$ & $682,415.00$ & $\begin{array}{l}8 \\
\% \\
\end{array}$ & $58,029.00$ & $663,773.00$ & $\begin{array}{l}9 \\
\% \\
\end{array}$ & $40,149.00$ & $\begin{array}{l}715,714.0 \\
0\end{array}$ & $\begin{array}{l}6 \\
\% \\
\end{array}$ & $37,597.00$ & $722,491.00$ & $\begin{array}{l}5 \\
\% \\
\end{array}$ & $77,549.00$ & $\begin{array}{l}786,663.0 \\
0\end{array}$ & $10 \%$ \\
\hline $\begin{array}{l}\text { CONSTR } \\
\text { UCTION/ } \\
\text { REAL } \\
\text { ESTATE }\end{array}$ & $\begin{array}{l}\text { ARBICO } \\
\text { PLC }\end{array}$ & $297,633.00$ & $\begin{array}{l}2,501,728.0 \\
0\end{array}$ & $\begin{array}{l}12 \\
\%\end{array}$ & $\begin{array}{l}- \\
252,299.00\end{array}$ & $\begin{array}{l}4,450,767.0 \\
0\end{array}$ & $\begin{array}{l}6 \\
\%\end{array}$ & $\begin{array}{l}341,722.0 \\
0\end{array}$ & $\begin{array}{l}4,532,183 . \\
00\end{array}$ & $\begin{array}{l}8 \\
\%\end{array}$ & $43,502.00$ & $\begin{array}{l}3,927,791.0 \\
0\end{array}$ & $\begin{array}{l}1 \\
\%\end{array}$ & $\begin{array}{l}107,178.0 \\
0\end{array}$ & $\begin{array}{l}5,351,996 . \\
00\end{array}$ & $2 \%$ \\
\hline & $\begin{array}{l}\text { JULIUS } \\
\text { BERGER } \\
\text { NIG PLC } \\
\end{array}$ & $\begin{array}{l}16,220,536 . \\
00\end{array}$ & $\begin{array}{l}227,261,257 \\
.00\end{array}$ & $\begin{array}{l}7 \\
\% \\
\end{array}$ & $\begin{array}{l}13,134,896 . \\
00\end{array}$ & $\begin{array}{l}256,045,781 \\
.00 \\
\end{array}$ & $\begin{array}{l}5 \\
\% \\
\end{array}$ & $\begin{array}{l}6,499,973 . \\
00\end{array}$ & $\begin{array}{l}245,086,2 \\
70.00\end{array}$ & $\begin{array}{l}3 \\
\% \\
\end{array}$ & $\begin{array}{l}- \\
1,498,029 . \\
00\end{array}$ & $\begin{array}{l}259,178,932 \\
.00\end{array}$ & $\begin{array}{l}- \\
1 \\
\%\end{array}$ & $\begin{array}{l}3,739,140 . \\
00\end{array}$ & $\begin{array}{l}275,393,7 \\
93.00 \\
\end{array}$ & $1 \%$ \\
\hline & $\begin{array}{l}\text { ROAADS } \\
\text { NIG PLC }\end{array}$ & $121,972.00$ & $909,067.00$ & $\begin{array}{l}13 \\
\%\end{array}$ & $138,294.00$ & $761,129.00$ & $\begin{array}{l}18 \\
\%\end{array}$ & $\begin{array}{l}134,841.0 \\
0\end{array}$ & $\begin{array}{l}641,356.0 \\
0\end{array}$ & $\begin{array}{l}21 \\
\%\end{array}$ & $\begin{array}{l}203,369.0 \\
0\end{array}$ & $538,203.00$ & $\begin{array}{l}38 \\
\%\end{array}$ & $\begin{array}{l}323,698.0 \\
0\end{array}$ & $\begin{array}{l}428,818.0 \\
0\end{array}$ & $-75 \%$ \\
\hline $\begin{array}{l}\text { CONGLO } \\
\text { MERATE } \\
\mathrm{S} \\
\end{array}$ & $\begin{array}{l}\text { A.G } \\
\text { LEVENTIS } \\
\text { NIG PLC } \\
\end{array}$ & 1037184 & 17546067 & $\begin{array}{l}6 \\
\% \\
\end{array}$ & $\begin{array}{l}1,227,392.0 \\
0\end{array}$ & $\begin{array}{l}14,712,124 . \\
00\end{array}$ & $\begin{array}{l}8 \\
\% \\
\end{array}$ & $\begin{array}{l}534,039.0 \\
0\end{array}$ & $\begin{array}{l}17,318,58 \\
6.00\end{array}$ & $\begin{array}{l}3 \\
\% \\
\end{array}$ & $\begin{array}{l}291,225.0 \\
0\end{array}$ & $\begin{array}{l}16,285,567 . \\
00\end{array}$ & $\begin{array}{l}2 \\
\%\end{array}$ & $\begin{array}{l}- \\
2,912,112 . \\
00\end{array}$ & $\begin{array}{l}13,943,27 \\
2.00\end{array}$ & $-21 \%$ \\
\hline & $\begin{array}{l}\text { JOHN HOLT } \\
\text { PLC }\end{array}$ & $264,000.00$ & $\begin{array}{l}9,258,000.0 \\
0\end{array}$ & $\begin{array}{l}3 \\
\% \\
\end{array}$ & $427,000.00$ & $\begin{array}{l}11,259,000 . \\
00\end{array}$ & $\begin{array}{l}4 \\
\% \\
\end{array}$ & $\begin{array}{l}- \\
171,000.0 \\
0\end{array}$ & $\begin{array}{l}10,921,00 \\
0.00\end{array}$ & $\begin{array}{l}- \\
2 \\
\%\end{array}$ & $\begin{array}{l}204,000.0 \\
0\end{array}$ & $\begin{array}{l}12,085,000 . \\
00\end{array}$ & $\begin{array}{l}2 \\
\%\end{array}$ & $\begin{array}{l}- \\
223,000.0 \\
0\end{array}$ & $\begin{array}{l}10,245,00 \\
0.00\end{array}$ & $-2 \%$ \\
\hline & $\begin{array}{l}\text { SCOA } \\
\text { NIGERIA } \\
\text { PLC }\end{array}$ & $144,906.00$ & $\begin{array}{l}8,083,645.0 \\
0\end{array}$ & $\begin{array}{l}2 \\
\%\end{array}$ & $88,444.00$ & $\begin{array}{l}10,247,444 . \\
00\end{array}$ & $\begin{array}{l}1 \\
\%\end{array}$ & $\begin{array}{l}- \\
1,255,526 \\
00\end{array}$ & $\begin{array}{l}10,848,76 \\
5.00\end{array}$ & $\begin{array}{l}- \\
12 \\
\%\end{array}$ & $\begin{array}{l}2,258,195 . \\
00\end{array}$ & $\begin{array}{l}14,137,648 . \\
00\end{array}$ & $\begin{array}{l}- \\
16 \\
\%\end{array}$ & $\begin{array}{l}- \\
1,897,170 . \\
00\end{array}$ & $\begin{array}{l}12,889,63 \\
0.00\end{array}$ & $-15 \%$ \\
\hline & $\begin{array}{l}\text { TRANSNAT } \\
\text { IONAL } \\
\text { CORPORAT } \\
\text { ION OF } \\
\text { NIGERIA } \\
\text { PLC } \\
\end{array}$ & $\begin{array}{l}9,032,151.0 \\
0\end{array}$ & $\begin{array}{l}149,464,413 \\
.00\end{array}$ & $\begin{array}{l}6 \\
\% \\
\end{array}$ & $\begin{array}{l}7,731,598.0 \\
0\end{array}$ & $\begin{array}{l}170,755,362 \\
.00\end{array}$ & $\begin{array}{l}5 \\
\% \\
\end{array}$ & $\begin{array}{l}3,319,529 . \\
00\end{array}$ & $\begin{array}{l}202,883,9 \\
49.00\end{array}$ & $\begin{array}{l}2 \\
\%\end{array}$ & $\begin{array}{l}5,928,348 . \\
00\end{array}$ & $\begin{array}{l}232,160,731 \\
.00\end{array}$ & $\begin{array}{l}3 \\
\%\end{array}$ & $\begin{array}{l}12,305,54 \\
7.00\end{array}$ & $\begin{array}{l}285,522,3 \\
04.00\end{array}$ & $4 \%$ \\
\hline & $\begin{array}{l}\text { UAC } \\
\text { NIGERIA } \\
\text { PLC } \\
\end{array}$ & $\begin{array}{l}13,935,016 . \\
00\end{array}$ & $\begin{array}{l}126,606,022 \\
.00\end{array}$ & $\begin{array}{l}11 \\
\% \\
\end{array}$ & $\begin{array}{l}14,096,932 . \\
00\end{array}$ & $\begin{array}{l}130,360,660 \\
.00\end{array}$ & $\begin{array}{l}11 \\
\% \\
\end{array}$ & $\begin{array}{l}7,733,077 . \\
00\end{array}$ & $\begin{array}{l}128,655,3 \\
28.00\end{array}$ & $\begin{array}{l}6 \\
\% \\
\end{array}$ & $\begin{array}{l}8,368,087 . \\
00\end{array}$ & $\begin{array}{l}138,229,559 \\
.00\end{array}$ & $\begin{array}{l}6 \\
\% \\
\end{array}$ & $\begin{array}{l}3,246,120 . \\
00\end{array}$ & $\begin{array}{l}130,617,1 \\
33.00\end{array}$ & $2 \%$ \\
\hline $\begin{array}{l}\text { AGRICUL } \\
\text { TURE }\end{array}$ & $\begin{array}{l}\text { FTN COCOA } \\
\text { PROCESSO } \\
\text { RS PLC }\end{array}$ & $\begin{array}{l}- \\
286,077.00\end{array}$ & $\begin{array}{l}4,553,277.0 \\
0\end{array}$ & $\begin{array}{l}- \\
6 \\
\%\end{array}$ & $\begin{array}{l}- \\
577,204.00\end{array}$ & $\begin{array}{l}4,421,423.0 \\
0\end{array}$ & $\begin{array}{l}- \\
13 \\
\%\end{array}$ & $\begin{array}{l}- \\
201,195.0 \\
0\end{array}$ & $\begin{array}{l}4,738,510 . \\
00\end{array}$ & $\begin{array}{l}- \\
4 \\
\%\end{array}$ & $\begin{array}{l}263,352.0 \\
0\end{array}$ & $\begin{array}{l}5,276,690.0 \\
0\end{array}$ & $\begin{array}{l}- \\
5 \\
\%\end{array}$ & $\begin{array}{l}- \\
419,730.0 \\
0\end{array}$ & $\begin{array}{l}5,004,460 . \\
00\end{array}$ & $-8 \%$ \\
\hline & $\begin{array}{l}\text { THE } \\
\text { OKOMU } \\
\text { OIL PALM } \\
\text { COMPANY } \\
\text { PLC } \\
\end{array}$ & $\begin{array}{l}2,687,301.0 \\
0 \\
\end{array}$ & $\begin{array}{l}15,035,022 . \\
00\end{array}$ & $\begin{array}{l}18 \\
\% \\
\end{array}$ & $\begin{array}{l}1,904,496.0 \\
0\end{array}$ & $\begin{array}{l}17,872,328 . \\
00\end{array}$ & $\begin{array}{l}11 \\
\% \\
\end{array}$ & $\begin{array}{l}2,898,645 . \\
00\end{array}$ & $\begin{array}{l}20,000,24 \\
0.00 \\
\end{array}$ & $\begin{array}{l}14 \\
\% \\
\end{array}$ & $\begin{array}{l}5,906,453 . \\
00\end{array}$ & $\begin{array}{l}24,507,665 . \\
00\end{array}$ & $\begin{array}{l}24 \\
\% \\
\end{array}$ & $\begin{array}{l}11,140,14 \\
2.00\end{array}$ & $\begin{array}{l}31,372,15 \\
2.00\end{array}$ & $36 \%$ \\
\hline & $\begin{array}{l}\text { PRESCO } \\
\text { PLC }\end{array}$ & $\begin{array}{l}2,333,970.0 \\
0\end{array}$ & $\begin{array}{l}32,663,299 . \\
00\end{array}$ & $\begin{array}{l}7 \\
\% \\
\end{array}$ & $\begin{array}{l}7,900,779.0 \\
0\end{array}$ & $\begin{array}{l}49,818,491 . \\
00\end{array}$ & $\begin{array}{l}16 \\
\%\end{array}$ & $\begin{array}{l}4,214,741 . \\
00\end{array}$ & $\begin{array}{l}55,477,99 \\
9.00\end{array}$ & $\begin{array}{l}8 \\
\%\end{array}$ & $\begin{array}{l}31,226,45 \\
2.00\end{array}$ & $\begin{array}{l}83,161,837 . \\
00\end{array}$ & $\begin{array}{l}38 \\
\%\end{array}$ & $\begin{array}{l}10,951,58 \\
1.00\end{array}$ & $\begin{array}{l}98,324,09 \\
5.00\end{array}$ & $11 \%$ \\
\hline
\end{tabular}




\begin{tabular}{|c|c|c|c|c|c|c|c|c|c|c|c|c|c|c|c|c|}
\hline & \begin{tabular}{|l} 
ELLAH \\
LAKES PLC
\end{tabular} & $25,384.00$ & $855,452.00$ & $\begin{array}{l}3 \\
\% \\
\end{array}$ & $31,232.00$ & $\begin{array}{l}1,129,957.0 \\
0\end{array}$ & $\begin{array}{l}3 \\
\% \\
\end{array}$ & $\begin{array}{l}- \\
24,861.00\end{array}$ & $\begin{array}{l}1,177,845 . \\
00\end{array}$ & $\begin{array}{l}- \\
2 \\
\%\end{array}$ & $\begin{array}{l}- \\
13,944.00\end{array}$ & $\begin{array}{l}1,195,441.0 \\
0\end{array}$ & $\begin{array}{l}- \\
1 \\
\%\end{array}$ & $\begin{array}{l}- \\
34,730.00\end{array}$ & $\begin{array}{l}1,180,872 . \\
00\end{array}$ & $-3 \%$ \\
\hline & $\begin{array}{l}\text { LIVESTOCK } \\
\text { FEEDS PLC } \\
\end{array}$ & $282,798.00$ & $\begin{array}{l}3,670,605.0 \\
0\end{array}$ & $\begin{array}{l}8 \\
\% \\
\end{array}$ & $402,151.00$ & $\begin{array}{l}5,752,787.0 \\
0\end{array}$ & $\begin{array}{l}7 \\
\% \\
\end{array}$ & $\begin{array}{l}300,115.0 \\
0\end{array}$ & $\begin{array}{l}4,569,513 . \\
00\end{array}$ & $\begin{array}{l}7 \\
\% \\
\end{array}$ & $\begin{array}{l}223,990.0 \\
0 \\
\end{array}$ & $\begin{array}{l}7,357,534.0 \\
0\end{array}$ & $\begin{array}{l}3 \\
\% \\
\end{array}$ & $\begin{array}{l}725,803.0 \\
0\end{array}$ & $\begin{array}{l}5,260,126 . \\
00\end{array}$ & $-14 \%$ \\
\hline \multirow[t]{8}{*}{$\begin{array}{l}\text { OIL AND } \\
\text { GAS }\end{array}$} & \begin{tabular}{|l} 
JAPAUL OIL \\
$\&$ \\
MARITIME \\
SERVICES \\
PLC \\
\end{tabular} & $460,274.00$ & $\begin{array}{l}38,776,602 . \\
00\end{array}$ & $\begin{array}{l}1 \\
\% \\
\end{array}$ & $\begin{array}{l}- \\
2,258,361.0 \\
0 \\
\end{array}$ & $\begin{array}{l}38,390,653 . \\
00\end{array}$ & $\begin{array}{l}- \\
6 \\
\% \\
\end{array}$ & $\begin{array}{l}- \\
7,899,057 . \\
00 \\
\end{array}$ & $\begin{array}{l}33,889,61 \\
8.00\end{array}$ & $\begin{array}{l}- \\
23 \\
\% \\
\end{array}$ & $\begin{array}{l}- \\
21,344,79 \\
7.00\end{array}$ & $\begin{array}{l}29,948,162 . \\
00\end{array}$ & $\begin{array}{l}- \\
71 \\
\% \\
\end{array}$ & $\begin{array}{l}- \\
13,081,59 \\
5.00\end{array}$ & $\begin{array}{l}28,001,56 \\
5.00\end{array}$ & $-47 \%$ \\
\hline & $\begin{array}{l}\text { OANDO } \\
\text { PLC } \\
\end{array}$ & $\begin{array}{l}7,711,850.0 \\
0\end{array}$ & $\begin{array}{l}556,707,119 \\
.00\end{array}$ & $\begin{array}{l}1 \\
\% \\
\end{array}$ & $\begin{array}{l}- \\
137,696,205 \\
.00\end{array}$ & $\begin{array}{l}892,353,671 \\
.00\end{array}$ & $\begin{array}{l}- \\
15 \\
\% \\
\end{array}$ & $\begin{array}{l}51,136,89 \\
8.00 \\
\end{array}$ & $\begin{array}{l}946,321,3 \\
09.00\end{array}$ & $\begin{array}{l}- \\
5 \\
\% \\
\end{array}$ & $\begin{array}{l}- \\
32,394,05 \\
4.00\end{array}$ & $\begin{array}{l}991,544,975 \\
.00\end{array}$ & $\begin{array}{l}- \\
3 \\
\% \\
\end{array}$ & $\begin{array}{l}27,068,14 \\
2.00 \\
\end{array}$ & $\begin{array}{l}1,040,175, \\
904.00\end{array}$ & $3 \%$ \\
\hline & $\begin{array}{l}\text { CONOIL } \\
\text { PLC }\end{array}$ & $\begin{array}{l}1,148,819.0 \\
0\end{array}$ & $\begin{array}{l}83,095,975 . \\
00\end{array}$ & $\begin{array}{l}1 \\
\%\end{array}$ & $\begin{array}{l}4,575,824.0 \\
0\end{array}$ & $\begin{array}{l}82,372,026 . \\
00\end{array}$ & $\begin{array}{l}6 \\
\% \\
\end{array}$ & $\begin{array}{l}1,532,174 . \\
00\end{array}$ & $\begin{array}{l}87,526,68 \\
7.00\end{array}$ & $\begin{array}{l}2 \\
\%\end{array}$ & $\begin{array}{l}3,448,397 . \\
00\end{array}$ & $\begin{array}{l}69,387,364 . \\
00\end{array}$ & $\begin{array}{l}5 \\
\%\end{array}$ & $\begin{array}{l}4,280,549 . \\
00\end{array}$ & $\begin{array}{l}69,833,46 \\
3.00\end{array}$ & $6 \%$ \\
\hline & $\begin{array}{l}\text { ETERNA } \\
\text { PLC } \\
\end{array}$ & $\begin{array}{l}1,069,428.0 \\
0\end{array}$ & $\begin{array}{l}18,253,144 . \\
00\end{array}$ & $\begin{array}{l}6 \\
\% \\
\end{array}$ & $\begin{array}{l}1,792,066.0 \\
0\end{array}$ & $\begin{array}{l}18,566,894 . \\
00\end{array}$ & $\begin{array}{l}10 \\
\% \\
\end{array}$ & $\begin{array}{l}1,306,585 . \\
00\end{array}$ & $\begin{array}{l}28,565,40 \\
9.00\end{array}$ & $\begin{array}{l}5 \\
\% \\
\end{array}$ & $\begin{array}{l}2,400,172 . \\
00\end{array}$ & $\begin{array}{l}31,690,081 . \\
00\end{array}$ & $\begin{array}{l}8 \\
\% \\
\end{array}$ & $\begin{array}{l}2,812,941 . \\
00\end{array}$ & $\begin{array}{l}48,045,73 \\
2.00 \\
\end{array}$ & $6 \%$ \\
\hline & $\begin{array}{l}\text { FORTE OIL } \\
\text { PLC }\end{array}$ & $\begin{array}{l}6,524,550.0 \\
0\end{array}$ & $\begin{array}{l}104,678,000 \\
.00\end{array}$ & $\begin{array}{l}6 \\
\%\end{array}$ & $\begin{array}{l}6,006,298.0 \\
0\end{array}$ & $\begin{array}{l}139,238,298 \\
.00\end{array}$ & $\begin{array}{l}4 \\
\%\end{array}$ & $\begin{array}{l}7,012,442 . \\
00\end{array}$ & $\begin{array}{l}121,757,9 \\
56.00\end{array}$ & $\begin{array}{l}6 \\
\%\end{array}$ & $\begin{array}{l}5,340,244 . \\
00\end{array}$ & $\begin{array}{l}140,756,492 \\
.00\end{array}$ & $\begin{array}{l}4 \\
\%\end{array}$ & $\begin{array}{l}10,627,15 \\
6.00\end{array}$ & $\begin{array}{l}147,237,8 \\
16.00\end{array}$ & $7 \%$ \\
\hline & $\begin{array}{l}11 \text { PLC } \\
\text { (FORMERL } \\
\text { Y MOBIL } \\
\text { OIL NIG } \\
\text { PLC) } \\
\end{array}$ & $\begin{array}{l}5,123,002.0 \\
0\end{array}$ & $\begin{array}{l}40,728,522 . \\
00\end{array}$ & $\begin{array}{l}13 \\
\% \\
\end{array}$ & $\begin{array}{l}8,446,137.0 \\
0\end{array}$ & $\begin{array}{l}49,226,575 . \\
00\end{array}$ & $\begin{array}{l}17 \\
\% \\
\end{array}$ & $\begin{array}{l}6,906,322 . \\
00\end{array}$ & $\begin{array}{l}54,072,08 \\
9.00\end{array}$ & $\begin{array}{l}13 \\
\% \\
\end{array}$ & $\begin{array}{l}12,019,89 \\
2.00\end{array}$ & $\begin{array}{l}61,701,328 . \\
00\end{array}$ & $\begin{array}{l}19 \\
\% \\
\end{array}$ & $\begin{array}{l}11,137,88 \\
6.00\end{array}$ & $\begin{array}{l}74,648,92 \\
8.00\end{array}$ & $15 \%$ \\
\hline & $\begin{array}{l}\text { MRS OIL } \\
\text { NIG PLC }\end{array}$ & $\begin{array}{l}1,407,143.0 \\
0\end{array}$ & $\begin{array}{l}65,694,626 . \\
00\end{array}$ & $\begin{array}{l}2 \\
\%\end{array}$ & $\begin{array}{l}1,282,053.0 \\
0\end{array}$ & $\begin{array}{l}57,846,626 . \\
00\end{array}$ & $\begin{array}{l}2 \\
\%\end{array}$ & $\begin{array}{l}1,460,843 . \\
00\end{array}$ & $\begin{array}{l}66,893,74 \\
1.00\end{array}$ & $\begin{array}{l}2 \\
\%\end{array}$ & $\begin{array}{l}2,287,347 . \\
00\end{array}$ & $\begin{array}{l}81,364,815 . \\
00\end{array}$ & $\begin{array}{l}3 \\
\% \\
\end{array}$ & $\begin{array}{l}996,609.0 \\
0\end{array}$ & $\begin{array}{l}62,190,31 \\
8.00\end{array}$ & $-2 \%$ \\
\hline & $\begin{array}{l}\text { TOTAL NIG } \\
\text { PLC }\end{array}$ & $\begin{array}{l}9,787,175.0 \\
0\end{array}$ & $\begin{array}{l}79,403,587 . \\
00\end{array}$ & $\begin{array}{l}12 \\
\%\end{array}$ & $\begin{array}{l}6,832,922.0 \\
0\end{array}$ & $\begin{array}{l}95,512,428 . \\
00\end{array}$ & $\begin{array}{l}7 \\
\% \\
\end{array}$ & $\begin{array}{l}6,495,390 . \\
00\end{array}$ & $\begin{array}{l}83,653,55 \\
5.00\end{array}$ & $\begin{array}{l}8 \\
\% \\
\end{array}$ & $\begin{array}{l}20,353,07 \\
6.00\end{array}$ & $\begin{array}{l}136,928,160 \\
.00\end{array}$ & $\begin{array}{l}15 \\
\%\end{array}$ & $\begin{array}{l}11,795,28 \\
3.00\end{array}$ & $\begin{array}{l}107,981,8 \\
73.00\end{array}$ & $11 \%$ \\
\hline
\end{tabular}

SOURCE: Financial Performance computed by the researcher from Nigerian Stock Exchange Factbbok 


\subsection{Analysis of the Research Question}

\subsection{DATA ANALYSIS}

Research Question 1. To what extent do manufacturing companies adopt Customer Accounting methods for financial improvement?

Table 4.1a. Manufacturing Companies' Scores on Customer Accounting Methods and Financial Improvement

\begin{tabular}{|c|c|c|c|c|c|c|c|c|}
\hline $\begin{array}{l}\text { Company } \\
\text { Code }\end{array}$ & $\begin{array}{l}\text { Financial } \\
\text { Performance (\%) }\end{array}$ & $\begin{array}{l}\text { CPA } \\
\text { Score }\end{array}$ & $\begin{array}{l}\text { LCPA } \\
\text { Score }\end{array}$ & $\begin{array}{l}\text { VCA } \\
\text { Score }\end{array}$ & $\begin{array}{l}\text { CPA } \\
\text { Adoption } \\
\text { level }\end{array}$ & $\begin{array}{l}\text { LCPA } \\
\text { Adoption } \\
\text { level }\end{array}$ & $\begin{array}{l}\text { VCA } \\
\text { Adoption } \\
\text { level }\end{array}$ & $\begin{array}{l}\text { CA Methods } \\
\text { Adoption }\end{array}$ \\
\hline A1 & 8.00 & 27.00 & 24.50 & 19.00 & 3.00 & 3.00 & 3.00 & 3.00 \\
\hline $\mathrm{A} 2$ & -4.20 & $\begin{array}{l}19.00 \\
\end{array}$ & 14.00 & 15.50 & 2.00 & 2.00 & 3.00 & 2.00 \\
\hline $\mathrm{A} 3$ & 5.00 & 30.00 & 23.25 & 21.25 & 3.00 & 3.00 & 3.00 & 3.00 \\
\hline A4 & 18.00 & 29.50 & 21.25 & 22.00 & 3.00 & 3.00 & 3.00 & 3.00 \\
\hline A5 & 8.20 & 23.66 & 21.33 & 17.00 & 3.00 & 3.00 & 3.00 & 3.00 \\
\hline A6 & 3.45 & 24.00 & 24.33 & 18.00 & 3.00 & 3.00 & 3.00 & 3.00 \\
\hline A7 & 6.40 & 28.75 & 25.25 & 21.25 & 3.00 & 3.00 & 3.00 & 3.00 \\
\hline A8 & 2.80 & 25.00 & 23.50 & 18.50 & 3.00 & 3.00 & 3.00 & 3.00 \\
\hline A9 & 23.40 & 27.00 & 24.00 & 19.50 & 3.00 & 3.00 & 3.00 & 3.00 \\
\hline A10 & 16.20 & 26.50 & 24.25 & 19.75 & 3.00 & 3.00 & 3.00 & 3.00 \\
\hline A11 & 21.59 & 25.25 & 25.75 & 22.25 & 3.00 & 3.00 & 3.00 & 3.00 \\
\hline A12 & 26.00 & 28.33 & 22.00 & 20.33 & 3.00 & 3.00 & 3.00 & 3.00 \\
\hline A13 & -3.00 & 24.67 & 21.33 & 18.00 & 3.00 & 3.00 & 3.00 & 3.00 \\
\hline A14 & 19.40 & $\begin{array}{l}17.67 \\
\end{array}$ & 16.67 & 18.00 & 2.00 & 2.00 & 3.00 & 2.00 \\
\hline A15 & 11.40 & 19.00 & 15.00 & 10.50 & 2.00 & 2.00 & 2.00 & 2.00 \\
\hline A16 & 6.40 & 31.00 & 26.00 & 22.50 & 3.00 & 3.00 & 3.00 & 3.00 \\
\hline A17 & 39.80 & 18.50 & 15.50 & 12.75 & 2.00 & 2.00 & 2.00 & 2.00 \\
\hline A18 & 62.80 & 31.00 & 26.75 & 22.25 & 3.00 & 3.00 & 3.00 & 3.00 \\
\hline A19 & 13.00 & 17.50 & 14.50 & 11.50 & 2.00 & 2.00 & 2.00 & 2.00 \\
\hline A20 & 2.00 & 14.25 & 12.00 & 9.75 & 2.00 & 2.00 & 1.00 & 2.00 \\
\hline A21 & -5.00 & 9.50 & 8.25 & 6.75 & 1.00 & 1.00 & 1.00 & 1.00 \\
\hline A22 & 5.80 & 15.50 & 13.00 & 11.00 & 2.00 & 2.00 & 2.00 & 2.00 \\
\hline A23 & 1.00 & 14.25 & 12.25 & 10.25 & 2.00 & 2.00 & 2.00 & 2.00 \\
\hline A24 & 10.80 & 8.25 & 7.00 & 6.00 & 1.00 & 1.00 & 1.00 & 1.00 \\
\hline $\mathrm{A} 25$ & 15.40 & 19.00 & 16.50 & 14.25 & 2.00 & 2.00 & 2.00 & 2.00 \\
\hline A26 & 12.40 & 20.75 & 17.75 & $\begin{array}{l}15.00 \\
\end{array}$ & 2.00 & 2.00 & 3.00 & 2.00 \\
\hline A27 & 7.60 & 17.25 & 15.00 & 12.50 & 2.00 & 2.00 & 2.00 & 2.00 \\
\hline A28 & 3.40 & 16.00 & 14.00 & 11.75 & 2.00 & 2.00 & 2.00 & 2.00 \\
\hline A29 & 3.00 & 16.50 & 14.50 & 12.25 & 2.00 & 2.00 & 2.00 & 2.00 \\
\hline A30 & 3.00 & 16.50 & 14.50 & 12.25 & 2.00 & 2.00 & 2.00 & 2.00 \\
\hline A31 & -.40 & 9.50 & 7.75 & 6.50 & 1.00 & 1.00 & 1.00 & 1.00 \\
\hline $\mathrm{A} 32$ & 1.00 & 15.00 & 13.00 & 10.75 & 2.00 & 2.00 & 2.00 & 2.00 \\
\hline A33 & -8.00 & $\begin{array}{l}10.00 \\
\end{array}$ & 8.00 & 6.50 & 1.00 & 1.00 & 1.00 & 1.00 \\
\hline A34 & 2.80 & 15.50 & 13.50 & 11.25 & 2.00 & 2.00 & 2.00 & 2.00 \\
\hline A35 & 7.20 & 16.50 & 14.50 & 12.25 & 2.00 & 2.00 & 2.00 & 2.00 \\
\hline A36 & $\begin{array}{l}-7.20 \\
\end{array}$ & $\begin{array}{l}10.00 \\
\end{array}$ & 8.25 & 6.75 & 1.00 & 1.00 & 1.00 & 1.00 \\
\hline A37 & 20.60 & 20.00 & 17.50 & 14.25 & 2.00 & 2.00 & 2.00 & 2.00 \\
\hline A38 & 16.00 & 16.50 & 14.50 & 12.25 & 2.00 & 2.00 & 2.00 & 2.00 \\
\hline A39 & .00 & 9.25 & 7.75 & 6.25 & 1.00 & 1.00 & 1.00 & 1.00 \\
\hline $\mathrm{A} 40$ & 2.20 & 15.25 & 13.25 & 11.00 & 2.00 & 2.00 & 2.00 & 2.00 \\
\hline A41 & 29.20 & 9.50 & 8.00 & 6.50 & 1.00 & 1.00 & 1.00 & 1.00 \\
\hline A42 & -3.80 & 9.00 & 7.00 & 5.75 & 1.00 & 1.00 & 1.00 & 1.00 \\
\hline $\mathrm{A} 43$ & 4.00 & 15.75 & 13.75 & 11.50 & 2.00 & 2.00 & 2.00 & 2.00 \\
\hline A44 & 7.00 & 15.25 & 13.25 & 11.00 & 2.00 & 2.00 & 2.00 & 2.00 \\
\hline A45 & 5.40 & 15.25 & 13.25 & 11.00 & 2.00 & 2.00 & 2.00 & 2.00 \\
\hline A46 & 15.40 & 18.25 & 16.00 & 13.25 & 2.00 & 2.00 & 2.00 & 2.00 \\
\hline A47 & 1.40 & 14.75 & 12.75 & 10.75 & 2.00 & 2.00 & 2.00 & 2.00 \\
\hline A48 & 10.60 & 15.25 & 13.25 & 11.00 & 2.00 & 2.00 & 2.00 & 2.00 \\
\hline
\end{tabular}

Source: Field Survey, 2018/2019Key: 1 = Non Adopters; 2 = Partial Adopters; 3 = Full Adopters

Table 4.1b. Extent of adoption of methods of Customer Accounting Methods by Manufacturing Companies for Financial Improvement

\begin{tabular}{|c|c|c|c|c|}
\hline & $\mathrm{N}$ & Mean & Std. Deviation & Remark \\
\hline Customer Profitability Analysis & 48 & 18.57 & 6.50 & Little Extent \\
\hline Life-time Customer Profitability Analysis & 48 & 16.02 & 5.78 & Little Extent \\
\hline Valuation of Customers as Assets & 48 & 13.54 & 5.04 & Little Extent \\
\hline
\end{tabular}


As shown in Tables 4.1a and 4.1b above, customer profitability analysis scale ranged from 7 to 35 andhas a scale average of 21. Companies' mean rating of their application of customer profitability analysis (CPA) was 18.57. This mean value is less than the scale average, it was decided that manufacturing companies to a little extent apply customer profitability analysis.

Companies' mean rating of their application of Life-time Customer Profitability Analysis (LCPA) was 16.02. Using a scale ranging from 6 to 30 and scale average of 18, their mean rating suggests that the extent of manufacturing companies' application of LCPA was little.
Likewise, the mean rating of the application of Valuation of Customers as Assets which was 13.54 was less than the scale average of 15.00 . Considering the scale ranging between 5 and 25, the scale mean of 15.00 and the actual mean rating by companies (Mean $=13.54$ ), it was concluded that companies also apply this method to a little extent

Using the categorizing schema stated in the chapter three, it was found that $16.7 \%$ of the companies fell within nonadopters of customer accounting methods, 54.2\% are partial adopters while $29.2 \%$ are full adopters. See table 4.2 shown below.

Table 4.2. Status of Adoption of customer accounting methods in Manufacturing Companies

$\begin{array}{lcc} & \text { Frequency } & \% \\ \text { Non Adopters } & 8 & 16.7 \\ \text { Partial Adopters } & 26 & 54.2 \\ \text { Full Adopters } & 14 & 29.2 \\ \text { Total } & 48 & 100.0\end{array}$

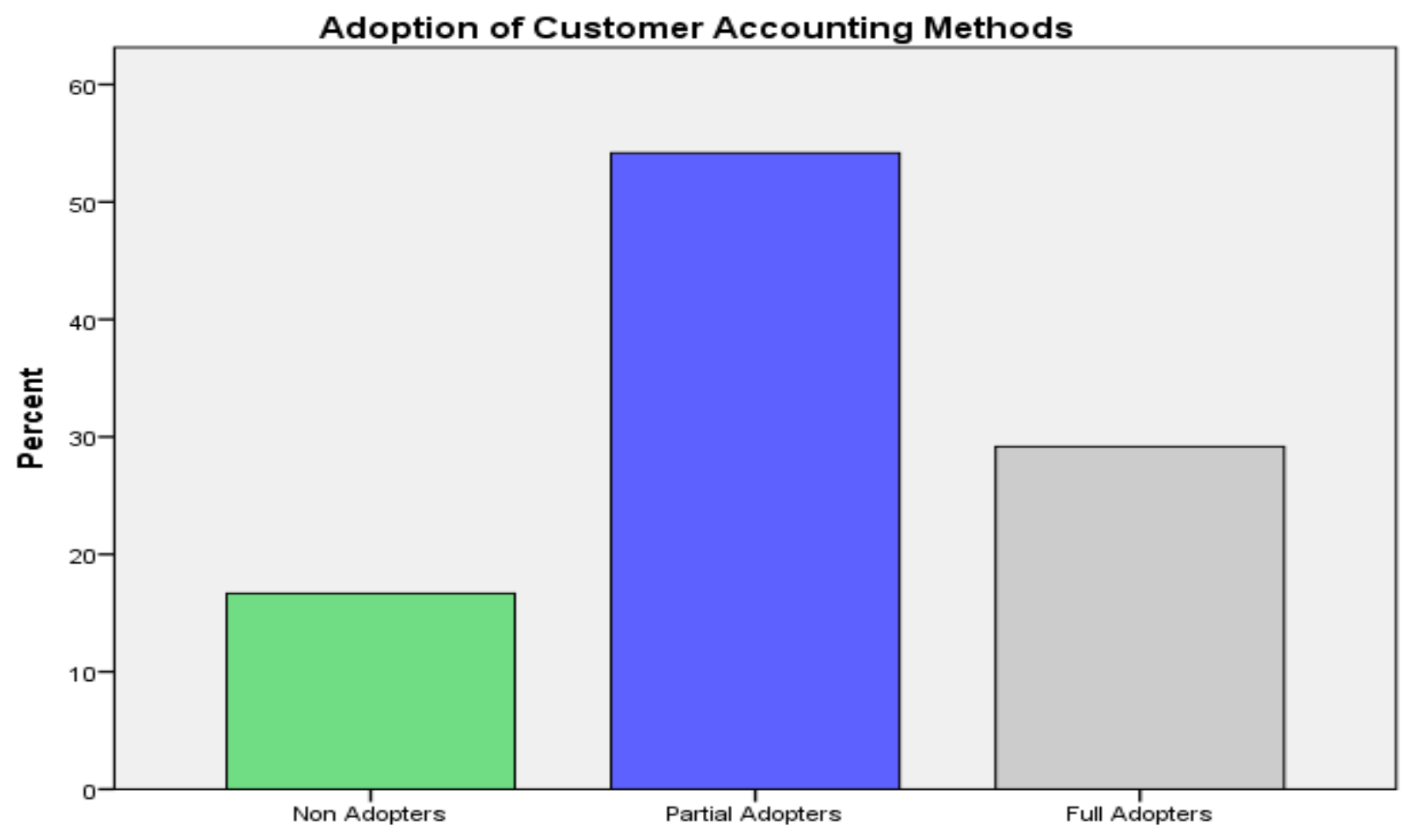

Adoption of Customer Accounting Methods

Fig. 4.1: Bar charts showing adoption of customer accounting methods by manufacturing companies

Research Question 2.To what extent do full adopters, partial adopters and none adopters of CA methods differ in financial performance?

Table 4.3. Mean Difference in Financial performance of Full adopters, Partial adopters and Non Adopters of Customer Accounting methods

N Mean SD

$\begin{array}{lc}\text { None Adopters } & 9 \\ \text { Partial Adopters } & 227 \\ \text { Full Adopters } & 17 \\ \text { Total } & 48\end{array}$

$\begin{array}{ccc}9 & 1.95 & 11.69 \\ 22 & 8.95 & 8.91 \\ 17 & 13.69 & 15.50 \\ 48 & 9.32 & 12.57\end{array}$


As shown in Table 4.3 above, the mean financial performance of full adopters of CA methods was 13.69, this was 4.74 greater than that of partial adopters with 8.95 and 11.74 greater than that of none adopters with 1.95 . This shows a small mean difference between full adopters and partial adopters and a large mean difference between full adopters and none adopters. However, the significance of this difference was tested using ANOVA as shown in table 4.4.

\subsection{Test of Hypothesis}

Hypothesis 1.Full adopters, partial adopters and non-adopters of Customer Accounting Methods differ significantly in financial performance.

Table 4.4: Analysis of Variance on the Difference between the Financial Performance of Full Adopters, Partial Adopters and Non-adopters of Customer Accounting Methods

\begin{tabular}{lccccc}
\hline & Sum of Squares & df & Mean Square & F & P-value \\
Between Groups & 1128.64 & 2 & 564.32 & 3.87 & .028 \\
Within Groups & 6548.56 & 45 & 145.52 & Sig. & \\
Total & 7677.20 & 47 & & \\
\hline
\end{tabular}

As shown in table 4.4, the F-ratio (df: 2/45) is 3.87 and the P-value (.028) is less than the stipulated 0.05 level of significance. It was therefore decided that there is a significant difference in financial performance of full adopters, partial adopters and none adopters of Customer Accounting methods. The null hypothesis was therefore supported.

Since the ANOVA test was significant, a scheffe Post Hoc test was carried out to identify where the significant difference existed. This is presented in Table 4.5 below.

Table 4.5: Scheffe Post-Hoc onthe difference between the financial performance of full and partial and none adopters of customer accounting methods
Adoption Level
Adoption Level
Mean Difference
Sig.

\begin{tabular}{llcc}
\hline \multirow{2}{*}{ Non Adopters } & Partial Adopters & -6.76 & .390 \\
& Full Adopters & $-14.42^{*}$ & .034 \\
Partial Adopters & Non Adopters & 6.76 & .390 \\
\multirow{2}{*}{ Full Adopters } & Full Adopters & -7.65 & .172 \\
& Non Adopters & $14.424^{*}$ & .034 \\
\hline
\end{tabular}

\section{*Significant}

As indicated by the Post-Hoc test (Scheffe test) in Table 4.5, there is a significant difference in the financial performance of non-adopters and full adopters of customer accounting methods. However, there is no significant difference between the financial performance of non-adopters and partial adopters as well as that of partial adopters and full adopters.

\section{i. $\quad$ Extent of application of CA methods}

\subsection{Discussion of Findings}

In the verge of ascertaining the extent of the application of CA methods in manufacturing companies in Nigeria, the researcher found out that the application rate of the three methods of Customer Accounting ( CPA, LCPA, VCA) is low (little extent). In the case of CPA, the results of the study indicate that customer profitability analysis with scale ranging from 7 to 35 and a scale average of 21, the companies' mean rating of their application was 18.57. While that of Life-time Customer Profitability Analysis (LCPA) was 16.02. Using a scale ranging from 6 to 30 and scale average of 18, this equally suggests that the extent of manufacturing companies' application of LCPA was little. In the same vein, the mean rating of the application of Valuation of Customers as Assets which was 13.54 was less than the scale average of 15.00. Considering the scale ranging between 5 and 25, the scale mean of 15.00 and the actual mean rating by companies (Mean $=13.54$ ), it was concluded that companies also apply this method to a little extent.

From the above, you see that the mean value of all the three CA methods is less than the scale average, which indicate that manufacturing companies to a little extent apply customer accounting methods. This agrees with Lord, Shanahan and Nolan (2007), in their study customer accounting technique usage was below the midpoint on a 7-point Likert scale, similar to that of Cadez (2006) who found that two out of three customer accounting techniques measured below the midpoint on a 7-point Likert scale. The reasons behind their findings may be explained by their research design, which used one single question to measure each customer accounting technique, which potentially created bias. There are also other strategic management accounting studies that contrasted the current study's findings like Al-mawali and Amoush, (2013), who found out that the extent of application of the three methods of customer accounting in Joranian context is moderate. The result further shows that out of the three CA dimensions, two dimensions scored mean values above the mid-point score of the 7-point likert scale of measurement. Customer profitability analysis was rated higher than the other two CA information dimensions, followed by lifetime customer profitability analysis and valuation of customers as assets were equally rated the lowest. 
$i$.

Difference in the financial performance of non, partial and full adopters

The study tried to find out if there exist any significant differences in the financial performance of the various categories of adopters of customer accounting methods. The study revealed that the mean financial performance of full adopters of CA methods was 13.69 , this was 4.74 greater than that of partial adopters with 8.95 and 11.74 greater than that of none adopters with 1.95. This shows a small mean difference between full adopters and partial adopters and a large mean difference between full adopters and none adopters

In the case of partial adopters when compared with non adopters, it was found out that partial adopter mean financial performance was 7.00 greater than that of non adopters and 4.74 less than full adopters as stated above. On the other hand non adopters mean financial performance was 7.00 less than partial adopters and 12.26 less than full adopters.

Analysis of the variance of the three categories of adopters shows the F-ratio (df: 2/45) is 3.87 and the P-value (.028) which is less than the stipulated 0.05 level of significance. It was therefore decided that there is a significant difference in financial performance of full adopters, partial adopters and none adopters of Customer Accounting methods.

\subsection{SUMMARY OF FINDINGS}

1. The result showed that the mean value of each of the three methods of customer accounting were less than the scale average. This means that the application of methods of Customer Accounting Methods by Manufacturing Companies for Financial Improvement were of little extent.

2. The mean financial performance of full adopters of CA methods was 13.69 , this was 4.74 greater than that of partial adopters with 8.95 and 11.74 greater than that of none adopters with 1.95. This shows a small mean difference between full adopters and partial adopters and a large mean difference between full adopters and none adopters. This indicates that a significant difference exist in the financial performance of full adopters, partial adopters and non-adopters of Customer accounting methods.

\subsection{CONCLUSION AND RECOMMENDATION}

Based on the findings it is obvious that customer accounting information usage have not receive much attention in Nigerian manufacturing companies, this is to say that CA is still at infant stageamong manufacturing companies in Nigeria. The extent of application here in Nigeria is little. It is imperative to say that once there is an improvement in the application and adoption of Customer accounting, financial performance will improve. This is because Customers accounting information provides leverage for achieving competitive advantage thereby leading to better performance. Heford (2008) assert that an organization that is effective in competitive intelligence obtains better and more complete information, enabling the organization to improve its decision-making. The partial and non-application of customer accounting by manufacturing companies is not helping them. The full application of customer accounting should be adopted. Standards can equally be set for determining the success factor of the application of customer accounting.

\section{REFERENCES}

[1] Al-Mawali, H., Zainuddin, Y., \& Ali, N. N. K. (2012).Customer accounting information usage and organizational performance. Business Strategy Series, 13(5), 215-223.

[2] Al-Mawali, H. \&Amoush A. (2013). Customer accounting information usage: A mixed-methodstudy. Interdisciplinary Journal of Contemporary Research in Business, Vol. 5 No.6

[3] Cadez, S. (2006). A cross industry comparison of strategic management accounting practices: An exploratory study.Economic and Business Review for Central and South-Eastern Europe, 8(3), 279-299.

[4] Central Bank of Nigeria (2013).Statement of Accounts and Annual reports. Abuja: CBN Chiekezie, N. R., Egbunike, P. A., \&Odum, A. N. (2014), Adoption of competitors focused accounting methods in selected Manufacturing Companies in Nigeria. Asian Journal of Economic Modelling, 2(3): $128-140$.

[5] Chiekezie, N. R. (2014). Competitor focused accounting and financial performance in selected listed Manufacturing firms in Nigeria. Ph.d Thesis.

[6] Day, G. S. (1994). The capabilities of market-driven organizations.The Journal of Marketing, 58(4), 37-52.

[7] Foster, G., \& Gupta, M. (1994). Marketing, cost management and management accounting.,6, 43-Journal of Management Accounting Research 77.

[8] Gale, B. T. \& Wood, R. C. (1994).Managing customer value. New York: Free Press.

[9] Guilding, C., McManus, L. (2002). The incidence, perceived merit and antecedents of customeraccounting: An exploratory note. Accounting, Organizations and Society, 27(1-2), 45-59.

[10] Hamzah, A. \& Tri-Dung, L. (2016).Customer Accounting and Environmental Uncertainty: Sequential Explanatory Study.International Review of Management and Marketing, 6(3), 532-543.available at http: www.econjournals.com

[11] Harande, Y. I. (2009). Information for industry in Nigeria, Accessed on 10/05/2018 from http:/digital commons.unl.edu/libphilpract/292

[12] Hesford, J. W. (2008). An empirical investigation of accounting information use in competitive intelligence. Journal of Competitive Intelligence and Management, 4(3), 17-49.

[13] Johnson, H.T. \& Kaplan, R.S. (1987). Relevance Lost: The Rise and Fall of Management Accounting, Boston: Harvard Business School Press.

[14] Levitt, T. (1983).After the Sale is Over, Harvard Business Review, 61, Sept/Oct, 87-93.

[15] Lord, B. R., Shanahan, Y. P., \& Nolan, B. M. (2007). The use and perceived merit of customeraccounting in New Zealand. Accounting Research Journal, 20(1), 47-59.

[16] Malinic, S., Jovanovic, D., \&Jankovic, S. (2012). Competitive management accounting: Response to the challenges of strategic business decision making. Economics and Organizations, 9 (3), 297-309.

[17] Malmi, T., Raulas, M. \&Sehm, J. (2004).An empirical study on customer profitability accounting, customer strategies and corporate performance, Paper presented at European Accounting Association Conference, Praque, 2004, 1 - 22.

[18] McManus, L. (2007). "The Construction of a Segmental Customer Profitability Analysis", Journal o fApplied Management Accounting Research, Volume 5, Number 2, pp. 59-74. 
[19] McManus, L., Guilding, C. (2008). Exploring the potential of customer accounting: A synthesis of the accounting and marketing literatures. Journal of Marketing Management, 24(7), 771-795.

[20] Muo, M.C. \&Okeke, T.C. (2009). Achieving vision 2020 in Nigeria: imperatives of economic diversification. Journal of the Management Sciences. $9(1), 162-177$.

[21] National Bureau of Statistics, 2017.

[22] Porter M., 1980, Competitive Strategy, Free Press, New York

[23] Smith Jr, C.W. (1993).A perspective on accounting-based debt covenant violations.Accounting Review, 289-303.

[24] Ward, K. (1992). Strategic Management Accounting. Oxford: Butterworth-Heineman.

[25] Woodruff, R. B. (1997). Customer value: The next source for competitive advantage. Journal of the academy of marketing science, 25(2), 139-153.

[26] Yonggui, W., \&Hing, P. L. (2004). Customer-focused performance and its key resource- baseddeterminants: An integrated framework. Journal of Global Competitiveness, 14(1/2), 34-59. 